\title{
A expectativa em relação ao treinamento influencia 0 impacto das ações de capacitação?*
}

\author{
Arquiléia Gonçalves** \\ Luciana Mourão***
}

\begin{abstract}
Sumário: 1. Introdução; 2. Treinamento, desenvolvimento e educação; 3. Principais modelos teóricos; 4. As variáveis estudadas; 5 . Pesquisas empíricas de avaliação de T\&D; 6. Método; 7. Resultados; 8. Discussão dos resultados; 9. Conclusão.
\end{abstract}

Summary: 1. Introduction; 2. Training, development and education; 3. Most important theoretical models; 4. Variables studied; 5. Empirical research assessment T\&D; 6. Method; 7. Results; 8. Discussion; 9. Conclusion.

Palavras-chave: avaliação de treinamento; expectativas de treinamento; impacto do treinamento; características dos treinandos.

KEY WORDs: trainig evaluation; training expectancy; impact of training; trainees characteristics.

O aumento da importância da área de treinamento e desenvolvimento para as organizações vem alimentando o interesse em investigar o impacto das ações de capacitação. Este estudo teve como principal objetivo identificar se características individuais influenciam o desempenho no trabalho das tarefas treinadas. A pesquisa, de natureza quantitativa, foi realizada em uma autarquia federal, com aplicação de questionários com escalas previamente validadas, em três momentos: antes do evento de capacitação (medição das expectativas em relação ao treinamento - 472

\footnotetext{
* Artigo recebido em nov. 2009 e aceito em set. 2010.

$*$ Mestre em psicologia. Universidade Salgado de Oliveira (Universo) e Comissão Nacional de Energia Nuclear (CNEN). Assistente em Ciência e Tecnologia. Endereço: Rua 8 de Dezembro, 264/1403 - Vila Isabel - CEP 20550-200, Rio de Janeiro, RJ, Brasil. E-mail: aipgon@yahoo. com.br.

$* * *$ Doutora em psicologia. Universidade Salgado de Oliveira (Universo) e Associação Internacional de Educação Continuada (Aiec). Professora do Mestrado em Psicologia. Endereço: Estrada Caetano Monteiro, 1833, Casa 13 - CEP 24320-570, Niterói, RJ, Brasil. E-mail: mourao.luciana@ gmail.com.
} 
questionários); imediatamente após o curso (avaliação da reação — 485 questionários); e quatro meses após o término do curso (avaliação do impacto do treinamento - 296 questionários). Os principais resultados apontam índices elevados para as expectativas em relação ao treinamento, sendo mais altos entre mulheres, pessoas com escolaridade mais baixa e ocupantes do cargo de assistente. Também foi alta a percepção do impacto do treinamento no trabalho, sobretudo entre os treinandos com escolaridade mais baixa, aqueles que participaram de treinamentos de natureza cognitiva, aqueles que tinham expectativas de melhoria além da performance e reações mais positivas. São discutidas implicações teóricas e práticas dos resultados encontrados e é apresentada sugestão de agenda de pesquisa.

Expectation on the training influences the impact of these actions? Expectations and impact of training activities

The importance of the development and training practices to the organizations is fuelling the interest in investigating the impact training actions. The main objective of this study is to identify if individual characteristics influence the performance of trained tasks at work. The research, of quantitative nature, was held in a federal autarchy with questionnaires application, with scales previously validated and in three phases: before the beginning of the training event (measure of expectancies related to the training - 472 questionnaires); immediately after the course (evaluation of the reaction - 485 questionnaires); and four months after the end of the course (evaluation of the impact of training - 296 questionnaires). The main results point to high ratings of expectations related to training, being higher among women, people with lower education and people who occupy assistant positions. The perception of impact of training at work was also high, in particular, among the trainees with lower education, who participated in training with cognitive nature, those who had improvement over performance expectancy and more positive reaction. The practical implications of the results are discussed and it is presented a suggested agenda to future researches.

\section{Introdução}

Há diferentes formas de aprendizagem e elas podem ser categorizadas em aprendizagem natural ou induzida. A aprendizagem ocorre no nível individual, com possíveis efeitos para as equipes e para a organização. Neste estudo será focada a área de treinamento e desenvolvimento (T\&D), uma das formas clássicas de aprendizagem induzida nas organizações.

A atividade de T\&D é hoje considerada um investimento crucial para as organizações. As ações de T\&D fazem parte do contexto organizacional de forma determinante, são influenciadas e influenciam as estratégias da organização. É necessário que o processo de T\&D esteja alinhado com as 
principais diretrizes corporativas e também com o ambiente no qual as empresas estão inseridas.

Não há dúvida de que as organizações têm utilizado mais treinamento como estratégia de recursos humanos (Campos et al., 2004). O acelerado avanço tecnológico tem provocado mudanças no sistema produtivo e aumentado a demanda cognitiva dos trabalhadores, caracterizando uma tendência de maior exigência em praticamente todos os postos de trabalho. Por conseguinte, as necessidades de aprendizagem no trabalho estão forçando uma transformação no perfil da área de $\mathrm{T} \& \mathrm{D}$, com a redução do tempo de interstício entre as ações de capacitação para cada empregado, com demandas de requalificação cada vez mais velozes.

As ações de capacitação precisam ser avaliadas para que os profissionais de T\&D possam conhecer os resultados dos cursos, principalmente, se os objetivos propostos foram alcançados. O crescente interesse das organizações por conhecer resultados de treinamento está diretamente associado ao aumento constante de pesquisas e fórmulas para tratamento dos dados observados nas ações de capacitação. Assim, é possível encontrar várias pesquisas, tabelas, fórmulas, conceitos, métodos e técnicas para aplicação nas avaliações de treinamento. Pode-se dizer que a etapa de avaliação de treinamento é o fim de um ciclo e o início de outro.

Tão importante como os demais estágios do processo de treinamento, a avaliação vai permitir principalmente que seus resultados revelem erros e acertos que ocorreram na aplicação das ações de capacitação e vai garantir a retroalimentação do sistema. Os modelos tradicionais de avaliação, entre eles o de Kirkpatrick (1976), contemplam as medidas de reação, aprendizagem, comportamento no cargo e resultados. No Brasil ainda se faz pouco uso da avaliação de impacto de T\&D, principalmente no nível dos resultados para a organização. Sem a avaliação de impacto, o profissional de T\&D não consegue saber o quanto o treinamento está sendo efetivo na mudança comportamental e nos desempenhos individual, das equipes ou da organização. Mas é preciso não só pesquisar se houve ou não impacto, como também conhecer suas variáveis preditoras, para que os profissionais de T\&D possam realizar intervenções. Nesse sentido é que se consideraram como possíveis variáveis explicativas do impacto do treinamento no trabalho a reação ao treinamento e a expectativa do treinando em relação às ações de capacitação.

A investigação de reação como variável preditora do impacto vem sendo feita por diversos pesquisadores, mas não há ainda consenso sobre a relação entre tais variáveis. Alguns autores encontraram uma relação significativa e 
positiva e outros não encontraram nenhuma relação — como será discutido na revisão de literatura.

Por outro lado, a investigação da expectativa em relação às ações de T\&D também vem sendo estudada, mas associada, na maioria das vezes, à motivação para o treinamento. O principal modelo adotado nesse tipo de pesquisa é o de Vroom (1964), que trabalha com um valor multiplicativo da valência, instrumentalidade e expectância. A valência significa recompensa, instrumentalidade se refere à estimativa de retorno de resultados e expectância considera o desempenho como caminho para obtenção de recompensa. A soma dessas três dimensões mediria a força motivacional do indivíduo (Lacerda, 2002). No presente estudo, a proposta é investigar apenas a dimensão da expectativa para verificar a possível contribuição da mesma no impacto do treinamento no trabalho. Porém, a expectativa estudada aqui não será focada no conteúdo do curso, mas sim na possível utilidade do mesmo no dia a dia de trabalho e em outras atividades que poderiam estar relacionadas aos treinamentos.

Os pesquisadores ainda não encontraram respostas definitivas para alguns questionamentos, tais como: o treinamento produz impacto no desempenho dos profissionais que são capacitados? Existe relação entre a expectativa que um participante tem em relação a um determinado curso e o resultado que este curso terá para essa pessoa (seja em nível de reação, seja em nível de impacto no comportamento no cargo)? Variáveis individuais - tais como sexo, idade, tempo de experiência, grau de escolaridade - influenciam nos resultados obtidos nas ações de capacitação? Essas são algumas questões que a presente pesquisa investigou.

Assim, o objetivo desta pesquisa foi identificar se características individuais, entre elas as expectativas em relação ao treinamento e reação, influenciam o impacto do treinamento no nível do comportamento no cargo.

\section{Treinamento, desenvolvimento e educação}

O subsistema de treinamento, desenvolvimento e educação pode ser compreendido como uma ciência (Salas e Cannon-Bowers, 2001) multidisciplinar que se apropria de conceitos e teorias de diversas disciplinas como pedagogia, administração, psicologia, sociologia, educação, aplicando de forma organizada e sistematizada essas teorias e esses conceitos em programas que visam influenciar o comportamento humano no trabalho. Os conceitos principais de TD\&E são formulados a partir das contribuições de métodos da pedagogia, 
estratégias da administração, conceitos da psicologia, teorias da sociologia e técnicas educacionais que, de forma harmônica, produzem uma nova área do conhecimento capaz de estudar mais profundamente o fenômeno aprendizagem induzida nas organizações.

As ações de T\&D compõem o conjunto de ações de aprendizagem induzida que ocorrem no ambiente organizacional. Nadler (1984), Wexley (1984), Latham (1988) e Goldstein (1991) deram importantes contribuições para definição de treinamento. Bastos (1991) fez uma análise das definições concluindo que Wexley enfatiza a ação planejada da organização, enquanto que Goldstein e Latham destacam o processo de aquisição/modificação de comportamentos voltado para melhorar o desempenho no trabalho. Já o nível desenvolvimento incorpora a expressão "desenvolvimento de recursos humanos", que foi utilizada pela primeira vez por Nadler (1984), para quem essa dimensão tem a função de promover aprendizagem nos empregados, visando ao alcance dos objetivos organizacionais. Sallorenzo (2000) entende desenvolvimento como um processo de aprendizagem mais geral que treinamento, porque propicia o amadurecimento de indivíduos de forma mais ampla, não específica para um posto de trabalho. Borges-Andrade (2002) salienta que a linha que divide os conceitos de T\&D está cada vez mais tênue, já que as organizações têm focado suas ações de capacitação em diferentes processos de aprendizagem que podem ser utilizados em vários contextos. Vargas e Abbad (2006) apresentam o conceito de TD\&E, onde a dimensão educação é incluída às dimensões anteriores, em função de cada vez mais as organizações estarem investindo em ações de educação formal para os seus funcionários. Contudo, neste estudo, será adotado o termo T\&D, pelo fato de a pesquisa investigar ações apenas nessas duas dimensões.

Conforme sinalizado por Borges-Andrade (2002), T\&D é um subsistema da área de gestão de pessoas. Assim, por ser parte de um sistema maior, interfere diretamente nas demais subáreas: recrutamento e seleção, benefícios, cargos e salários, qualidade de vida, pagamento e avaliação de desempenho, fornecendo subsídios para aplicação de políticas adequadas. A área de T\&D tem função de destaque na harmonia do sistema de gestão de pessoas, contribuindo para que os demais subsistemas alcancem seus objetivos e metas. Ou seja, se a área de seleção preenche uma vaga, se um empregado é promovido ou se o subsistema de avaliação identifica pontos frágeis no seu desempenho, provavelmente a área de T\&D será acionada para complementar as ações deflagradas e contribuir para o sucesso da tomada de decisão. 
Há um consenso de que a área de treinamento tem sido considerada estratégica para as organizações (Borges-Andrade, 2002; Salas e CannonBowers, 2001; Vargas e Abbad, 2006). Contudo, a pesquisa de Campos e colaboradores (2004), realizada em empresas paulistas de médio e grande porte, sinaliza que, no Brasil, apesar de ter aumentado a importância da área de treinamento, ela ainda não tem os dados sistematizados. As autoras relatam em sua pesquisa que faltam informações sobre a proporção entre salários e treinamento, o número de dias de treinamento realizados por funcionário, entre outras. $\mathrm{O}$ estudo faz um paralelo com pesquisas internacionais e mostra que há no cenário nacional, assim como no exterior, maior importância atribuída ao treinamento pelas organizações. Os participantes da pesquisa apontam que houve um aumento nos investimentos em treinamento nos últimos três anos. Segundo as autoras, as empresas estão se preocupando com as tendências do mercado que prevê destaque para as funções ligadas à gestão estratégica, à informática e à qualidade, apontando para um futuro promissor para a área de T\&D.

No Brasil, Borges-Andrade e Oliveira-Castro (1996) realizaram uma revisão das pesquisas produzidas, entre 1980 e 1993, sobre avaliação de treinamento, desenvolvimento e educação nas organizações. No artigo, os autores descreveram a produção nacional na área de TD\&E e identificaram algumas lacunas. A partir dessas lacunas, propuseram uma agenda de pesquisa para a área, em que duas merecem destaque por estarem relacionadas ao presente projeto: (a) investigação das teorias da expectância, da equidade e de autorrealização e nos conceitos de eficácia do treinamento baseada na interação entre características da clientela e tipos de competências; e (b) criação de modelos preditivos, integrando os diversos níveis de avaliação, bem como criação de instrumentos para coleta de dados.

Salas e Cannon-Bowers (2001) descreveram e analisaram o progresso dos estudos sobre treinamento em cinco áreas de pesquisa, entre elas a de avaliação do treinamento. No texto dos autores fica evidente o interesse dos pesquisadores na etapa de avaliação. A tabela 1 apresenta a relação de algumas pesquisas analisadas pelos autores e os resultados encontrados.

Pode-se observar que a avaliação de treinamento teve lugar de destaque nas pesquisas e cada pesquisador apresentou uma nova maneira de observar ou de diagnosticar a sua importância e seu inter-relacionamento com as demais etapas do processo de T\&D. Uma das conclusões da revisão de Salas e Cannon-Bowers (2001) refere-se à relevância da transferência da aprendizagem na explicação dos modelos de efetividade das ações de capacitação nas organizações. 
Tabela 1

Pesquisas encontradas por Salas e Cannon-Bowers (2001) que estudaram avaliação de treinamento

\begin{tabular}{|ll|}
\hline Pesquisas & Resultados \\
\hline Tannembaun e cols. (1993) & Esboçaram as condições pré e durante \\
& o treinamento que podem influenciar na \\
Korendizagem e os fatores que podem facilitar na & transferência das habilidades aprendidas. \\
& Discutiram a importância de caracterizar fatores e \\
processos em que intervenções do treinamento \\
são executadas e transferidas nas organizações. \\
Kozlowski e cols. (2000) & Consideraram os fatores que influenciam a eficácia \\
& da transferência vertical nas ações de treinamento. \\
Kraiger e cols. (1993) & Forneceram novos conceitos de aprendizagem, de \\
& avaliação e medidas. \\
Ford e cols. (1997) & Aproveitaram "oportunidade de executar" como \\
uma maneira de compreender a transferência de & treinamento. \\
Cannon-Bowers e Salas (1995) & Desenvolveram um modelo para compreender \\
& o clima para transferência nas organizações e as \\
condições que a realçam. \\
Cannon-Bowers e cols. (1998) \\
Avançaram demonstrando circunstâncias, conceitos \\
e intervenções que podem realçar a prática.
\end{tabular}

\section{Principais modelos teóricos}

Dos modelos teóricos de avaliação de TD\&E, serão destacados neste artigo dois estrangeiros (Kirkpatrick, 1976; Alvarez, Salas e Garofano, 2004) e um nacional (Borges-Andrade, 1982). A revisão de literatura realizada por Salas e Cannon-Bowers (2001) aponta que o modelo de Kirkpatrick (1976) continua sendo o mais popular em avaliação de treinamento. Entretanto, trabalhos recentes vêm melhorando esses instrumentos de avaliação. Kirkpatrick (1976) defende que a avaliação de treinamento deve contemplar as medidas de reação, aprendizagem, comportamento no cargo e resultados. O autor compreendia que os níveis de avaliação mantêm entre si forte relacionamento positivo, ou seja, que a reação seria preditora da aprendizagem, que por sua vez seria preditora do impacto no comportamento no cargo e este preditor de resultados. Alliger e Janak (1989) consideraram que o modelo de Kirkpatrick poderia induzir ao entendimento de que o nível de resultados é melhor que os demais, o que seria um equívoco. Os mesmos autores discordam da inferên- 
cia de causalidade entre as avaliações de reação e aprendizagem, pois esses autores encontraram correlações negativas entre esses dois primeiros níveis. No Brasil, outros autores como Abbad, Gama e Borges-Anadrade (2000) também não confirmaram o relacionamento dos três primeiros níveis. Os autores alertam que, embora ainda sejam muito utilizados nas pesquisas estrangeiras, estudos mais recentes têm identificado problemas na articulação dos quatro níveis do modelo.

Alvarez, Salas e Garofano (2004) construíram o Modelo Integrado de Avaliação e Efetividade de Treinamento (IMTEE). A construção do IMTEE foi baseada numa revisão de literatura no período de 1993 a 2002. No estudo, os autores definem com clareza as diferenças entre avaliação e efetividade de treinamento e fornecem aos especialistas valiosa ferramenta que identifica os diversos inter-relacionamentos existentes - e nem sempre visíveis - entre variáveis e características de um programa de treinamento.

O IMTEE mensura quatro níveis de avaliação: (a) análise de necessidades, subdividida em conteúdo e projeto do treinamento, mudanças nos treinandos e demandas organizacionais; (b) reações; (c) transferência; e (d) resultados. A análise do modelo permite verificar que alguns níveis se interrelacionam formando uma cadeia e constroem um sistema, no qual os resultados dos diversos níveis dependem uns dos outros. De acordo com o IMTEE, os resultados do nível reação dependeriam apenas das características individuais dos treinandos. As mudanças nos treinandos, autoeficácia pós-treinamento, aprendizagem cognitiva e desempenho têm como possíveis preditoras as características individuais e características do treinamento. O modelo também relata a influência de características individuais, do treinamento e organizacionais sobre a transferência do treinamento e os resultados.

Ainda, analisando o modelo, podem ser observados outros inter-relacionamentos entre as variáveis: autoeficácia pós-treinamento influencia e é influenciada por aprendizagem cognitiva e desempenho do treinando; e desempenho do treinando influencia transferência que, por sua vez, influencia resultados.

O IMTEE permite um avanço em relação ao modelo de Kirkpatrick (1976), incluindo novos níveis bem como alterando sua hierarquia. O modelo proposto por Alvarez, Salas e Garofano (2004) amplia a visão de que avaliação de treinamento consiste numa etapa isolada de um programa de treinamento, oferecendo subsídios para que os gestores de recursos humanos possam vislumbrar saídas para corrigir erros e realçar os pontos que obtiveram sucesso.

No Brasil, Borges-Andrade (1982) identificou outras variáveis que interferem e que são importantes na avaliação dos programas de T\&D. O Modelo 
de Avaliação Integrado e Somativo (MAIS), proposto pelo autor, acrescenta variáveis do ambiente e de processo como indicativos de interferência nos resultados da ação de capacitação. O MAIS propõe cinco componentes (insumos, procedimentos, processos, disseminação e ambiente) como preditores de outros componentes (resultados imediatos e efeitos em longo prazo).

\section{As variáveis estudadas}

\section{Expectativa}

Em linguagem comum, o termo expectativa significa "esperança fundada em supostos direitos, probabilidades ou promessas" (Dicionário Aurélio). Ou seja, expectativas podem estar associadas a desejos e crenças que antecipam acontecimento futuro. Em psicologia, expectativa é definida como "uma atitude tensa e um tanto emocional para uma perspectiva de certo acontecimento — sinal, antecipação que realça a preparação motora, previsão ou premeditação e o aspecto intelectual" (Comprehensive dictionary of psychological terms). Assim, pode-se dizer que um grau maior ou menor de expectativa que o indivíduo tenha sobre determinado evento contribuiria para o resultado de seu planejamento.

Considerando o modelo MAIS, de Borges-Andrade (1982), expectativa estaria compreendida no componente insumos que o autor define como fatores físicos e sociais e estados comportamentais, geralmente associados ao treinando, anteriores ao treinamento e que podem afetar sua realização. Acredita-se que expectativa pode estar relacionada à forma como o evento foi disseminado na organização. Neste estudo, expectativa para treinamento é definida como uma atitude prévia à realização do evento de capacitação, que permite ao treinando um ajustamento às experiências que ele irá vivenciar.

\section{Reação}

O termo reação é muito rico em significado e definições e seu entendimento está muito relacionado ao contexto e ao objeto de estudo. Como significado geral, de uso comum, reação é definida como "resposta a uma ação qualquer por meio de outra ação que tende a anular a precedente" (Dicionário Aurélio). A psicologia dá mais atenção ao significado do termo e incorpora fatores que podem influenciar no seu uso. Na definição geral dada por esta 
ciência, reação é a "atividade de um organismo que se dá em resposta a fator externo ou interno identificável. [...] Pode tratar-se de resposta a um reflexo, a uma resposta condicionada, a uma resposta emocional ou a uma alteração no sistema imunológico em resposta a fatores afetivos" (Dicionário de psicologia, Roland Doron e Françoise Parot). Assim, nas definições apresentadas pode-se observar que, independentemente do objeto de estudo, reação está sempre associada a estímulo/resposta. O construto reação tem sido objeto de estudo de pesquisadores da área de T\&D, que veem a possibilidade de compreenderem resultados de treinamento a partir das reações apresentadas pelos participantes após os eventos de capacitação. O estudo da reação dos participantes faz parte dos modelos de avaliação de treinamento de Kirkpatrick (1976) e de Alvarez, Salas e Garofano (2004). A relação entre reação e os demais níveis - inclusive impacto do treinamento no comportamento no cargo - vêm sendo objeto de estudo em pesquisas brasileiras e estrangeiras, mas sem consenso sobre o poder preditivo de tal variável. No presente estudo, foi utilizada a definição de avaliação de reação de Abbad e colaboradores (2000:26): "o nível de satisfação dos participantes com a programação, o apoio ao desenvolvimento do curso, a aplicabilidade, a utilidade e os resultados de treinamento".

\section{Impacto do treinamento no trabalho}

Impacto em linguagem comum significa "metido à força, impelido"; impelir significa "impulsionar para algum lugar, empurrar, arremessar" (Dicionário Aurélio). Também foi feita pesquisa do termo "impacto" em dicionários de psicologia, recursos humanos e psicologia social e em nenhum deles o termo é contemplado. A definição atribuída ao termo em linguagem comum (dicionários de língua portuguesa) ignora intensidade e direção e estabelece movimento como condição fundamental. Mas o significado do termo impacto, quando aplicado a fenômenos científicos, exige um complemento de informações acerca da natureza desse movimento no que diz respeito tanto à intensidade quanto à direção.

O impacto do treinamento no trabalho corresponde a resultados obtidos no treinamento e que são posteriormente aplicados no trabalho. Esses resultados podem ocorrer em três níveis: indivíduo (desempenho no cargo ou comportamento no cargo), equipe e organização (esses dois últimos compreendendo a mudança organizacional e o valor final). Um aspecto a ser considerado é o de que, em última instância, a principal razão de se realizar ações de 
T\&D nas organizações é justamente a busca de uma melhoria no desempenho, seja essa melhoria imediata ou em médio e longo prazo.

O impacto no nível do comportamento no cargo focaliza o resultado do treinamento no desempenho do treinando no seu contexto de trabalho e constitui o primeiro indicador visível de resultados para os profissionais que não estão diretamente envolvidos com as ações de T\&D. O impacto do treinamento pode também ser explicado como a aquisição e aplicação das atitudes esperadas no trabalho. Várias pesquisas, nacionais e estrangeiras, têm buscado encontrar preditores para o impacto do treinamento no nível do comportamento no cargo (Abbad et al., 2000; Freitas e Borges-Andrade, 2004; Ford et al., 1992; Lacerda, 2002; Meneses, 2002; Rowold, 2007; Tziner et al., 2007; Velada et al., 2007; Warr e Bunce, 1995). Os resultados indicam que algumas variáveis individuais ajudam a explicar a aplicação dos conhecimentos, habilidades e atitudes aprendidos nos eventos de capacitação. Porém, as variáveis com maior poder de predição pertencem ao contexto, ou seja, são variáveis situacionais. Para este estudo, optou-se pela definição de impacto de treinamento como sendo: "corresponde ao efeito do treinamento sobre o desempenho, a motivação e/ou atitudes da clientela" (Abbad, 1999:38).

\section{Pesquisas empíricas de avaliação de T\&D}

Nesta seção serão apresentados alguns relatos de pesquisas estrangeiras e nacionais sobre avaliação de eventos de TD\&E, que tiveram como variável critério diferentes níveis de resultados de ações de TD\&E e como variáveis preditoras características da clientela, características do treinamento e variáveis contextuais (relativas ao nível organizacional).

Ford e colaboradores (1992) pesquisaram as oportunidades para desempenhar as tarefas treinadas como preditoras para a transferência dos conteúdos aprendidos num programa de treinamento. Na pesquisa realizada com 180 universitários da Força Aérea dos Estados Unidos, por meio da aplicação de questionários aos treinandos e a seus supervisores, os pesquisadores encontraram que as dimensões características organizacionais, contexto do trabalho e características individuais são relacionadas quando o indivíduo transfere para o trabalho o que aprendeu no treinamento. Os achados de Ford e colaboradores (1992) confirmam a hipótese de que oportunidades para desempenhar tarefas estão mais relacionadas a contexto de trabalho e a características individuais. Indivíduos com autoeficácia mais elevada têm maior probabilidade de desempenhar a maioria das tarefas mais complexas e difíceis. Indivíduos 
que possuem alta habilidade cognitiva tiveram resultados mais positivos. No presente estudo, assim como foi feito por Ford e colaboradores, também se investigou a influência de características individuais sobre uma possível transferência para o trabalho do que foi aprendido no treinamento, embora com variáveis distintas das que foram estudadas por esses autores.

Warr e Bunce (1995) também investigaram os resultados de ações de treinamento, porém focaram sua pesquisa na influência das características do treinando sobre os resultados da aprendizagem aberta. A pesquisa empírica realizada pelos autores avaliou 106 gerentes juniores, matriculados em um programa de treinamento de uma empresa britânica, e teve entre os seus achados associação negativa entre idade e aprendizagem; maior utilização pelos treinandos de estratégias de aprendizagem analítica que comportamental; e associação positiva da aprendizagem com a motivação, autoeficácia, qualificações educacionais e experiência na função. O estudo de Warr e Bunce (1995), assim como esse estudo, investigou os relacionamentos das variáveis idade e tempo de experiência com a avaliação de ações de T\&D. Porém, na pesquisa aqui proposta, o foco não será na aprendizagem, mas sim no impacto do treinamento no comportamento no cargo. Outro aspecto similar entre a presente pesquisa e a realizada por esses autores está na utilização da variável reação do treinando.

Abbad e colaboradores (2000) investigaram o relacionamento existente entre três dos mais tradicionais níveis de avaliação: reação, aprendizagem e impacto do treinamento no trabalho. Os autores validaram uma escala para avaliação de reação ao treinamento, que corresponde à escala utilizada no presente estudo. Como principais resultados do estudo, os autores apontam o forte relacionamento observado entre as variáveis de reações com os demais níveis. Ainda, encontraram correlação significativa e positiva entre reação e impacto do treinamento no trabalho, mas afirmam que o impacto do treinamento no trabalho nem sempre estará correlacionado com resultados de aprendizagem. O trabalho de Abbad e colaboradores (2000) acrescenta à presente pesquisa dados importantes acerca dos relacionamentos entre os níveis de avaliação do treinamento. Os achados dos autores questionam os pressupostos do modelo de Kirkpatrick (1976) e revelam que os relacionamentos entre os níveis de aprendizagem, reações e impacto ainda representam uma incógnita para os pesquisadores. Isso demonstra a importância da presente pesquisa para acrescentar novos resultados que confirmem ou não os encontrados por esses autores.

Meneses (2002) pesquisou o relacionamento de características dos treinamentos e de sua clientela (autoeficácia, lócus de controle, motivação e características demográficas), suporte à transferência e impacto do treinamento 
no trabalho. $\mathrm{O}$ autor utilizou uma amostra de 536 sujeitos funcionários de três organizações dos setores financeiro, telefônico e aeroportuário do Distrito Federal. Como principais resultados o autor encontrou que: (a) suporte psicossocial percebido pelos participantes do treinamento e autoeficácia contribuíram significativamente para a explicação de impacto do treinamento no trabalho; (b) suporte psicossocial percebido pelas chefias dos participantes do treinamento e quantidade de instrutores por turma contribuíram significativamente para a explicação da heteroavaliação do impacto do treinamento no trabalho; (c) entre as variáveis relativas a características da clientela, autoeficácia foi a única que explicou uma porção significativa das respostas de impacto. $\mathrm{O}$ trabalho de Meneses (2002) evidencia apenas uma variável de característica da clientela no modelo explicativo do impacto do treinamento no trabalho, mas fica a possibilidade de investigação de outras variáveis, tais como a expectativa em relação ao treinamento que foi alvo da presente pesquisa.

Lacerda (2002) pesquisou o relacionamento entre características dos treinandos (motivação para aprender, motivação para transferir e valor instrumental do treinamento), reações e suporte à transferência, como variáveis antecedentes do impacto do treinamento no trabalho. A pesquisa foi feita com a aplicação de questionários em 284 participantes de 28 cursos do Tribunal de Contas da União. A autora se baseou na teoria da expectância de Vroom (1964) - modelo multiplicativo, ou seja: valência $\times$ instrumentalidade $\times$ expectância. Os resultados gerais da pesquisa de Lacerda (2002) apontam que impacto do treinamento no trabalho foi explicado a partir de três variáveis: suporte psicossocial, valor instrumental do treinamento e reação ao desempenho do instrutor. Os resultados apresentados por Lacerda (2002) de que a motivação instrumental e a reação ao desempenho do instrutor são preditores do impacto do treinamento no trabalho constituem importante reflexão para a presente pesquisa. A motivação instrumental pode estar relacionada à expectativa do treinando em relação ao curso e, além disso, a variável reação, objeto de estudo de Lacerda (2002), também foi contemplada no modelo desta pesquisa.

Freitas e Borges-Andrade (2004) investigaram as relações existentes entre atitudes dos treinandos e transferência do treinamento a partir de uma pesquisa com treinandos de vários cursos realizados em um banco público. Para Freitas e Borges-Andrade (2004), o levantamento de dados acerca de crenças de treinamento contribui para a realização de diversas intervenções que permitam a ampliação das possibilidades de aplicação dos treinamentos, como a elaboração de desenho de programas, bem como relacionar características individuais à efetividade do treinamento. A comprovação da associa- 
ção entre crenças e transferência da aprendizagem, encontrada por Freitas e Borges-Andrade (2004), permite às organizações intervirem nessa variável para potencializar os resultados do treinamento. Essa pesquisa tem grande importância para este estudo porque é provável que as crenças sobre o sistema de T\&D influenciem a expectativa que os funcionários têm em relação aos treinamentos para os quais foram designados ou escolheram participar.

Outro estudo nessa área foi realizado por Rowold (2007), que pesquisou o impacto das expectativas dos treinandos, reações ao treinamento, cumprimento das expectativas e comprometimento com a organização como possíveis variáveis preditoras da efetividade do treinamento. A pesquisa foi feita com 130 empregados de dez centrais de atendimento de uma empresa alemã. A categoria características da clientela, foco da pesquisa de Rowold, foi escolhida por três razões principais: o aumento do interesse dos pesquisadores nas diferenças individuais em outras áreas de investigação; o fato de as características individuais terem relevância no processo de seleção dos treinandos; o fato de as atitudes e a motivação para aprender dos treinandos poderem influenciar na efetividade do treinamento. Como principais resultados, o estudo revelou que: (a) treinandos com níveis mais altos de escolaridade apresentaram maior conhecimento declarado após o curso; (b) motivação para aprender teve efeito positivo sobre a quantidade declarada de conhecimento adquirido; (c) reação ao treinamento (satisfação) e percepção de utilidade do treinamento não mostraram relação com os níveis de conhecimento declarado; (d) cumprimento de expectativas teve impacto positivo sobre aquisição de conhecimento declarado. Além desses resultados, o estudo também demonstrou que nível educacional tem relação negativa com expectativas pré-treinamento e cumprimento de expectativas pós-treinamento. A pesquisa de Rowold (2007) também contribuiu sobremaneira para o presente estudo por investigar o efeito das expectativas dos treinandos sobre os resultados do treinamento.

Tziner e colaboradores (2007) estudaram seis características individuais de trabalhadores (conscienciosidade, autoeficácia, motivação para aprender, orientação para aprendizagem, orientação para desempenho e instrumentalidade) e uma característica do ambiente de trabalho (clima para transferência do treinamento) como possíveis preditoras da aprendizagem e do impacto do treinamento no comportamento no cargo. A pesquisa foi feita com 130 empregados que participaram de um programa de treinamento para manutenção e atualização de conhecimentos técnico-profissionais em uma indústria de energia. Como principal resultado, Tziner e colaboradores (2007) encontraram que características de personalidade do treinando (conscienciosidade, auto- 
eficácia, motivação para aprender, orientação para aprendizagem, orientação para desempenho e instrumentalidade) e a característica ambiental clima para transferência do treinamento têm relacionamento positivo com os resultados de aprendizagem e de desempenho no cargo. Outro resultado que merece destaque é o fato de indivíduos com maior grau de formação escolar terem melhores resultados na avaliação de desempenho no cargo e também maior percepção sobre as oportunidades de aplicação das habilidades aprendidas no ambiente de trabalho. O estudo de Tziner e colaboradores (2007) contribui com a presente pesquisa por também investigar variáveis individuais que predizem resultados da efetividade do treinamento, destacando-se o uso comum nos dois estudos do grau de escolaridade e do impacto no nível do desempenho no cargo.

Velada e colaboradores (2007) estudaram fatores que determinam a transferência do treinamento para o contexto do trabalho, considerando 182 funcionários de uma rede de supermercados. A pesquisa analisou o poder de predição sobre a transferência do treinamento de três conjuntos de variáveis: características individuais dos treinandos, características do treinamento e características do ambiente de trabalho. Os resultados indicaram que todas as variáveis independentes tiveram relacionamento significativo e positivo com a variável transferência do treinamento. Os autores sugerem novas pesquisas que investiguem fatores pré-treinamento como possíveis preditores da transferência do treinamento para o trabalho. Tal sugestão foi incorporada ao presente estudo, onde se investigou se as expectativas pré-treinamento influenciam na posterior transferência do que foi aprendido.

\section{Método}

Considerando a revisão de literatura da área e as lacunas apresentadas pelos pesquisadores do tema, a presente pesquisa partiu da hipótese central de que expectativas da clientela, características demográficas e funcionais da clientela, características do treinamento e reação ao treinamento são variáveis preditoras da avaliação de treinamento no nível do impacto no comportamento no cargo, esperando-se que pessoas de escolaridade mais baixa, com expectativas e reação mais positivas, que participaram de cursos de natureza cognitiva e com menos tempo de serviço percebam maior nível de impacto do treinamento.

Esta pesquisa foi realizada em uma autarquia federal, com sede no Rio de Janeiro e institutos de pesquisa em São Paulo, Belo Horizonte, Recife e Rio de Janeiro, além de escritórios em diversas cidades. O presente estudo 
configura a primeira experiência da organização com aplicação de questionários para mensurar o impacto do treinamento no trabalho, constituindo uma oportunidade de desenvolver uma nova cultura de avaliação de treinamento.

O conjunto de ações de capacitação que foram avaliadas compreendeu 23 cursos, distribuídos em 39 turmas. Os cursos foram realizados na sede e nas unidades localizadas no Rio de Janeiro, em São Paulo e Belo Horizonte. Dos 23 cursos, 19 tiveram carga horária de 24 horas e os demais tiveram 8, 12, 16 e 40 horas. Com relação ao conteúdo, 58,5\% foi comportamental e 41,5\% cognitivo. Os cursos avaliados foram ministrados por instrutores externos à organização, por meio de duas modalidades de contratação: licitação e escolha da empresa pela área interessada.

Os instrumentos utilizados são autoaplicáveis, tendo sido utilizados três questionários: (a) o primeiro questionário, antes do evento de capacitação, com o objetivo de avaliar o grau de expectativa dos participantes com relação à atividade de treinamento da qual iriam participar. Esse instrumento foi construído pela pesquisadora tomando por base o instrumento de impacto de treinamento desenvolvido e validado por Abbad (1999) e revalidado por Pilati e Abbad (2005); (b) o segundo questionário, aplicado imediatamente após o término do evento de capacitação, teve como objetivo avaliar a reação do treinando ao curso. Esse instrumento já validado foi utilizado por diversos pesquisadores no Brasil (Meneses, 2002; Lacerda, 2002; Abbad, 1999); e (c) o terceiro questionário, aplicado entre 90 e 120 dias após o evento de capacitação, teve como objetivo avaliar o impacto do treinamento no trabalho. Esse último instrumento foi criado por Abbad (1999) e adaptado por Pilati e Abbad (2005).

O tratamento dos dados foi feito através do Software SPSS, versão 15.0, com as seguintes análises estatísticas: (a) limpeza do banco de dados; (b) análise de componentes principais/análise fatorial, para a validação da escala de expectativas pré-treinamento; (c) análises descritivas; (d) correlação; (e) Teste T; (f) Anova; e (g) regressão múltipla.

O questionário de expectativa de treinamento foi desenvolvido neste estudo e baseou-se na escala de impacto do treinamento no trabalho desenvolvido e validado por Abbad (1999) e revalidado por Pilati e Abbad (2005). $\mathrm{O}$ instrumento que serviu de base para a construção da escala de expectativa media a percepção do treinando acerca do quanto os conteúdos ensinados no curso estavam sendo utilizados nas tarefas do trabalho. Porém, aventou-se a hipótese de que diferentes graus de expectativas em relação às ações de T\&D poderiam provocar maior ou menor percepção de impacto do treinamento. Então, adotou-se as mesmas ações avaliadas no impacto, alterando o tempo 
do verbo do passado (utilizo, com frequência, em meu trabalho atual, o que foi ensinado no curso) para o futuro (acredito que vou utilizar, com frequência, em meu trabalho atual, o que será ensinado no treinamento). A escala de expectativa contém 10 itens, dois a menos que a escala de impacto. Isso ocorreu porque a "escala modelo" continha um item que se referia à ação que não pode ser transformada do passado para o futuro (recordo-me). O segundo item (Minha participação nesse curso aumentou minha autoconfiança) foi desconsiderado por ser muito semelhante a outro item da escala e pelo fato de Pilati e Abbad (2005), na revalidação da escala, sugerirem sua exclusão. Os dez itens finais da escala estão associados a uma escala tipo Likert, variando de 1 , para discordo fortemente, a 5 , concordo fortemente. Esse questionário foi aplicado antes do início do curso, quando os treinandos já se encontravam no ambiente de treinamento.

Para a mensuração da reação ao treinamento foi utilizada a escala completa de avaliação de reação aos cursos desenvolvida por Abbad e colaboradores (2000). Essa escala possui 37 itens e mensura aspectos diversos relativos aos treinamentos como: programação, aplicabilidade, utilidade e desempenho do instrutor. O instrumento adota uma escala tipo Likert, a qual mensura cinco alternativas de respostas: ótimo (5), muito bom (4), bom (3), regular (2), ruim (1). A escala de reação ao treinamento utilizada foi validada psicometricamente por Abbad e colaboradores (2000), em uma amostra de aproximadamente 4 mil servidores públicos, participantes de 229 cursos de treinamento. Os autores trabalham com a possibilidade de três fatores, mas indicam a utilização de apenas dois: reações aos resultados, aplicabilidade e expectativas de suporte; e reação à programação e ao apoio organizacional. Os coeficientes de fidedignidade (Alfa de Cronbach) reportados para esses fatores foram 0,95 e 0,92 , respectivamente, enquanto o Alfa de Cronbach da escala no presente estudo foi de 0,98.

No presente estudo, não foram utilizados os mesmos fatores sugeridos por Abbad e colaboradores (2000), pois se encontrou uma correlação de Pearson alta e positiva $(r=0,81, \mathrm{p}<0,001)$ entre os dois fatores. Em função disso, realizou-se nova análise fatorial, a qual indicou a extração de apenas um fator, tanto pelo critério do eigenvalue maior que 1 , como pelo critério da análise paralela (considerando 485 questionários e 37 itens), pois apenas um fator possui eigenvalue empírico superior ao eigenvalue aleatório (Enzmann, 1997).

Considerando os itens da escala de Abbad e colaboradores (2000), a análise da escala para a amostra atual também sugeriu a manutenção de todos os itens, pois qualquer item que fosse retirado não acarretaria acréscimo ao Alpha de Cronbach, que foi de 0,98. Assim, a variável reação figurou na regres- 
são com um único fator. Este questionário foi aplicado imediatamente após o término do curso, ainda no ambiente de sala de aula.

O questionário sobre o impacto do treinamento no trabalho, validado por Abbad (1999), é composto de 12 itens associados a uma escala tipo $L i$ kert de cinco pontos, variando de 1 , para discordo fortemente, a 5, concordo fortemente. Porém, Pilati e Abbad (2005), na análise confirmatória da escala, sugeriram a supressão de dois itens, com conteúdos redundantes. Assim, a escala de impacto sugerida pelos autores contém 10 itens e estrutura unifatorial. O questionário se propõe a medir ações deflagradas após a realização de cursos de capacitação, com foco no impacto produzido pelo treinamento no desempenho individual no trabalho (p. ex.: "Utilizo com frequência em meu trabalho atual o que foi ensinado no curso."; "A qualidade do meu trabalho melhorou nas atividades diretamente relacionadas ao conteúdo do curso."). Os coeficientes de fidedignidade (Alfa de Cronbach) reportados para a escala foram 0,90 (no estudo original) e 0,92 (no presente estudo).

Como o objetivo foi mensurar o impacto do treinamento, a aplicação dessa escala precisou ser feita um tempo após a realização do mesmo para que fosse possível medir seu efeito. No caso do presente estudo, o questionário de impacto foi aplicado num período de 90 a 120 dias após a realização do curso.

\section{Resultados}

Para o tratamento dos dados, procedeu-se às seguintes ações: limpeza do banco de dados, retirada de dados faltosos e outliers; análise de componentes principais/análise fatorial para a validação da escala de expectativas pré-treinamento e para confirmação da escala de reação; análises descritivas dos escores dessas escalas e de seus fatores; correlações bivariadas entre as variáveis dependentes e independentes; Teste t, Anova; e regressão múltipla para checagem das hipóteses de pesquisa.

A amostra da presente pesquisa foi estimada em 300 questionários pareados relativamente a três momentos de coleta de dados, sendo um instrumento de expectativas em relação à $\mathrm{T} \& \mathrm{D}$, outro de avaliação de reação ao treinamento e o último de impacto do treinamento no trabalho. Como havia necessidade de parear os instrumentos de coleta de dados e a taxa de retorno de cada um deles foi diferente, a amostra total foi ampliada e ficou com 492 sujeitos. Assim, foi possível parear 290 questionários de pessoas que responderam ao questionário de expectativa de treinamento e também ao de impacto 
ou que responderam ao de reação e ao de impacto. O conjunto que respondeu aos três instrumentos de coleta de dados correspondeu a 287 questionários.

A quantidade de casos omissos, considerando o conjunto dos instrumentos, foi de cerca de $2 \%$, tendo sido feita, nesse caso, a opção de substituição pela média. Em relação à variável impacto, optou-se por não considerar as respostas dos sujeitos que não responderam a esta escala, uma vez que $o$ percentual de casos faltosos ficou acima de $5 \%$, não sendo, portanto, recomendada a substituição pela média. Também foram excluídos do banco de dados os sujeitos que responderam a apenas um dos instrumentos de coleta de dados, pois sem o pareamento dos dados não é possível realizar as análises bivariadas.

Após a retirada dos outliers uni e multivariados, considerando-se os parâmetros da Distância Mahalanobis e a análise dos boxplot, o banco de dados, que continha 463 sujeitos, ficou com 419 casos válidos. Em relação à normalidade verificou-se que, entre as variáveis, apenas impacto do treinamento no trabalho apresentou níveis adequados de skewness e kurtosis, segundo os parâmetros de Tabachnick e Fidell (1996). As escalas de expectativa e a de reação tiveram de passar por transformações para ficar com indicadores adequados de normalidade.

Os resultados da análise descritiva da variável dependente mostram que houve uma percepção de impacto relativamente alta dos cursos realizados $(\mathrm{M}=3,79$; $\mathrm{DP}=0,66)$, o que indica uma percepção dos treinandos de que houve uma aplicação no trabalho da ordem de $75,8 \%$ do que foi ensinado nos cursos de capacitação avaliados. Ou seja, os funcionários percebem que estão conseguindo transferir para o dia a dia do trabalho os conteúdos aprendidos, de forma a melhorar seu desempenho na realização das tarefas.

Em relação à variável expectativas do treinamento, foi desenvolvida e validada uma escala com base nos itens do instrumento de impacto do treinamento no trabalho (criado e validado por Abbad, 1999). A escala refere-se às expectativas dos funcionários acerca da melhoria que o treinamento vai promover no seu trabalho e em atividades não diretamente relacionadas a ele. Com a finalidade de escolher a estrutura empírica mais adequada aos dados, foram realizadas: a análise do número de eigenvalues acima de um e das variâncias explicadas, a análise do scree plot, a análise paralela e a análise da consistência teórica. Em relação aos eigenvalues, apenas dois ficaram acima de um, explicando $64,8 \%$ da variância total das respostas aos itens. Para confirmar o número de fatores a serem extraídos, realizou-se também a análise paralela, proposta por Enzmann (1997), a qual apresenta os valores aleatórios de eigenvalues para uma matriz de dados com 10 itens e 415 sujeitos. De acordo com esse teste, devem 
ser extraídos tantos fatores quantos os eigenvalues empíricos forem superiores aos eigenvalues aleatórios. A análise dos dados apontou dois eigenvalues empíricos superiores aos eigenvalues aleatórios. Assim, a indicação final foi a de dois fatores, a saber: Fator 1: Expectativa de utilidade e melhoria de performance e Fator 2: Expectativa de melhoria além da performance. No que se refere à correlação entre os dois fatores da escala, encontrou-se um coeficiente de Pearson elevado $(0,65)$. Porém, mesmo com essa alta correlação, optou-se por trabalhar com dois fatores por ser a estrutura com maior compatibilidade teórica e também por ser a clara indicação dos testes estatísticos de análise fatorial, análise de componentes principais e análise paralela. Nenhum item mostrou-se complexo e as cargas fatoriais foram bastante elevadas, sugerindo que não houve ambiguidade no processo de separação dos itens. Assim, o Fator 1, que mensura as expectativas de utilidade e melhoria de performance, ficou com cinco 5 itens com cargas fatoriais variando de 0,69 a 0,79 e um Alpha de Cronbach igual a 0,89 . O Fator 2 , que mensura as expectativas de melhoria além da performance, também ficou com cinco itens, com cargas fatoriais variando de 0,68 a 0,74 e um Alpha de Cronbach de 0,87. Em relação à análise das médias, observou-se que elas foram mais elevadas $(\mathrm{m}=4,51$; $\mathrm{DP}=0,55)$ para o Fator 1 (Expectativa de utilidade e melhoria de performance), embora também tenham sido bastante altas $(\mathrm{m}=4,22$; $\mathrm{DP}=0,67)$ para o Fator 2 (Expectativa de melhorias além da performance). $\mathrm{O}$ teste $\mathrm{t}(\mathrm{p} \leq 0,05)$ para amostras pareadas revelou que há diferenças significativas entre essas médias.

Para verificar quais variáveis contribuem para explicar o impacto do treinamento, realizou-se uma análise de regressão padrão, tendo como variáveis independentes as expectativas da clientela, escolaridade, o tempo de trabalho, a natureza do treinamento e a reação ao treinamento sobre o impacto do treinamento no trabalho. Porém, antes de incluir tais variáveis no modelo, realizou-se uma análise de multicolinearidade entre as variáveis independentes, a qual indicou alta correlação entre os dois fatores de expectativas em relação às ações de T\&D e os dois fatores de reação ao treinamento.

Em relação à variável expectativas da clientela, em função da alta correlação entre seus dois fatores foi feito um teste de multicolinearidade. Os valores de VIF $(1,61)$ e de tolerância $(0,62)$ no teste de colinearidade indicaram que as variáveis independentes relativas aos dois fatores de expectativa, embora com uma correlação alta $(0,61)$, não apresentaram problemas de colinearidade (Myers, 1992; Menard, 1995). Da mesma forma, no mesmo teste, a análise da proporção de variância dos valores do eigenvalues e do condition index não indica problemas de multicolinearidade, sinalizando que, para o presente estudo, os dois fatores poderiam ser incluídos em um único modelo empírico. 
Os resultados das estatísticas descritivas da variável reação ao treinamento apresentaram escores médios elevados $(\mathrm{M}=4,28$ e $\mathrm{DP}=0,71$, para uma escala de 1 a 5 , na qual quanto mais próximo de 5 mais favorável é a avaliação). Isso sinaliza que os treinandos ficaram satisfeitos com os treinamentos realizados no que diz respeito aos itens relativos ao planejamento e condução dos treinamentos. Os escores mínimo $(1,45)$ e máximo $(5,0)$ apontaram amplitude de resultados com alguns insatisfeitos e outros bastante satisfeitos com os cursos.

Em relação à variável características do treinamento, foram categorizados dois tipos, de acordo com sua natureza: (a) comportamental; e (b) cognitivo. As variáveis incluídas como independentes foram: escolaridade, tempo de trabalho, natureza do treinamento, expectativas da clientela e reação ao treinamento. Os resultados da regressão mostram que todas as variáveis previstas permaneceram no modelo, exceto as expectativas de utilidade e melhoria de performance e o tempo de trabalho. A variância explicada pelo modelo foi de $24 \%\left(R^{2}\right.$ ajustado $\left.=0,24\right)$. As variáveis que apresentaram maiores valores de Beta foram escolaridade $(\beta=-0,27$; $\mathrm{p}<0,001)$ e expectativa de melhoria além da performance $(\beta=0,23$; $\mathrm{p}<0,001)$. Reação $(\beta=0,17$; $\mathrm{p}<0,003)$ e natureza do treinamento $(\beta=0,12 ; \mathrm{p}<0,03)$ também contribuíram para a explicação do modelo, conforme pode ser visto na tabela 2 .

Tabela 2

Regressão com a VD impacto do treinamento no trabalho

\begin{tabular}{|lccc|}
\hline Variáveis & B & $\beta$ & sr $^{2}$ \\
\hline Escolaridade & $-0,299^{* *}$ & 0,273 & 0,047 \\
Tempo de trabalho & $-0,030$ & $-0,058$ & 0,029 \\
Expectativa de melhoria da performance & 0,210 & 0,139 & 0,111 \\
Expectativa de melhoria além da performance & $0,323^{* *}$ & 0,230 & 0,100 \\
Natureza do treinamento & $0,165^{*}$ & 0,122 & 0,078 \\
Reação ao treinamento & $0,229^{* *}$ & 0,169 & 0,076 \\
\hline Média $=3,79 \quad \mathrm{DP}=0,66 \quad \mathrm{~F}=16,37$ & $\mathrm{P}<0,001$ & \\
\hline $\mathrm{R}=0,50 \quad \mathrm{R}^{2}=0,25$ & & \\
\hline$* \mathrm{P}<0,05 ; \mathrm{P}<0,01$ & & \\
\hline
\end{tabular}

Esses resultados indicam que, quanto menor a escolaridade, maior o impacto do treinamento no trabalho. Isso demonstra que, em geral, os funcionários de nível médio que foram capacitados tiveram melhor percepção da aplicação no trabalho dos conteúdos aprendidos do que os funcionários de níveis mais elevados. 
Por outro lado, as pessoas que têm expectativas mais elevadas em relação à melhoria além da performance apresentaram maior percepção do impacto do treinamento no seu desempenho. Ou seja, os treinandos que, antes de realizar o treinamento, acreditavam que o mesmo lhes possibilitaria melhorias que vão além da aplicação direta no trabalho tenderam a perceber uma melhoria de performance mais acentuada.

A variável natureza do treinamento, que também figurou no modelo final, indicou que sujeitos que participaram de cursos de natureza cognitiva tenderam a perceber maior impacto desses cursos no seu trabalho do que sujeitos que participaram de cursos de natureza comportamental. Pode-se, portanto, dizer que foi confirmada a hipótese de que os cursos de natureza cognitiva tendem a contribuir mais para o impacto do treinamento que os cursos de natureza comportamental.

Por fim, a regressão indicou que, quanto mais positiva a avaliação de reação ao treinamento, mais positiva tende a ser também a avaliação do impacto do treinamento. Em outras palavras, quem ficou satisfeito com o curso apresentou, alguns meses depois, uma percepção maior de que foi possível transferir para o trabalho os conteúdos aprendidos e que, portanto, o treinamento influenciou positivamente o seu desempenho.

\section{Discussão dos resultados}

A hipótese norteadora desta pesquisa afirmava que pessoas com escolaridade mais baixa, com expectativa e reação mais positivas, que participaram de cursos de natureza cognitiva e com menos tempo de serviço apresentam nível de impacto do treinamento mais elevado. $O$ resultado deste estudo aponta para uma alta percepção de impacto dos conteúdos ensinados no treinamento sobre o trabalho. Esse resultado sugere que o programa de capacitação oferecido pela instituição correspondeu às necessidades dos funcionários e atendeu à demanda de conhecimento exigida pelo trabalho. O impacto do treinamento é um dos níveis mais importantes a ser alcançado e um dos mais valorizados pelas chefias. É por meio desse resultado que os supervisores avaliam o sucesso ou o fracasso do treinamento, razão pela qual têm crescido o interesse nesse tipo de mensuração (Borges-Andrade e Oliveira-Castro, 1996; Borges-Andrade, 2002; Salas e Cannon-Bowers, 2001).

Esse resultado favorável provoca na organização e, principalmente, nas chefias atitudes mais favoráveis em relação ao sistema de T\&D, o que possibilita maior comprometimento com as atividades de treinamento e, 
consequentemente, maior facilidade na liberação de funcionários para participarem dos cursos.

A relação negativa entre as variáveis escolaridade e impacto, encontrada no estudo, confirma a hipótese formulada de que indivíduos com menor escolaridade percebem maior impacto do treinamento no trabalho e pode ser atribuída ao fato de os funcionários de menor escolaridade terem menos chances fora da instituição de verem suas necessidades de conhecimento e saber atendidas. Assim, esses funcionários veem na organização a oportunidade de ampliar seus conhecimentos e melhorar seu desenvolvimento intelectual. É possível que a fórmula encontrada por aqueles que têm menor escolaridade seja a de imprimir maior esforço tanto para aplicar os conhecimentos aprendidos como para buscar oportunidades de aplicação. Outro ponto que pode ter contribuído para esse resultado refere-se ao fato de os funcionários com maior escolaridade desenvolverem um espírito mais crítico na sua avaliação; é bom salientar que, de acordo com Abbad (1999), essas avaliações são perceptuais e com conteúdo eminentemente subjetivo.

Warr e Bunce (1995) também pesquisaram a importância das qualificações educacionais para os resultados do treinamento, porém adotaram como variável dependente a aprendizagem. Esses autores encontraram relacionamento positivo entre a variável qualificações educacionais e a aprendizagem. Assim, os resultados do presente estudo vão de encontro ao de Warr e Bunce (1995), pois, enquanto os autores apontam que a aprendizagem está positivamente relacionada com a maior qualificação educacional, nesta pesquisa encontrou-se que quem tem menor escolaridade tende a aproveitar melhor os cursos realizados (percepção de impacto mais elevada). Porém, é possível que haja uma diferença entre esses dois níveis de resultados do treinamento, isto é, a escolaridade pode contribuir para a aprendizagem e não contribuir para a percepção do impacto do treinamento no trabalho. Nesse sentido, Alliger e Janak (1989) e Abbad e colaboradores (2000) encontraram que impacto nem sempre estará correlacionado com resultados de aprendizagem.

Outro estudo que investigou o relacionamento de variável similar à escolaridade - habilidade cognitiva - com impacto do treinamento foi o de Ford e colaboradores (1992). Esses autores encontraram que indivíduos com alta habilidade cognitiva apresentam resultados mais positivos no nível impacto do treinamento no cargo. Assim como Ford e colaboradores, Tziner e colaboradores (2007) também encontraram resultados que se contrapõem aos da presente pesquisa para a variável escolaridade. No estudo desses autores, treinandos com maior escolaridade apresentaram melhores resultados no impacto do treinamento no trabalho. Uma possível diferença para 
os resultados encontrados talvez resida nas realidades distintas dos países onde os estudos foram realizados, pois no Brasil o acesso ao ensino superior ainda é muito restrito. Assim, quem tem menor escolaridade depende mais das oportunidades oferecidas pelas organizações, o que pode explicar uma maior percepção de aproveitamento dos cursos.

Expectativa de melhoria além da performance apresentou-se como preditora do impacto do treinamento no trabalho; esse resultado pode estar relacionado ao fato de as pessoas com expectativas mais positivas estarem mais abertas à adoção de novos caminhos para execução de suas tarefas e, assim, produzirem melhores oportunidades para reforçar o aprendizado. Velada e colaboradores (2007) e Meneses (2002) indicaram que autoeficácia apresentou relacionamento positivo com transferência do treinamento. Autoeficácia pode ser descrito como um construto que favorece uma melhor expectativa e, assim, o presente estudo reforçaria os achados desses autores. Já Rowold (2007) apontou que as expectativas pré-treinamento não explicam o conhecimento declarado após o curso. Ou seja, para o autor, expectativa pré-treinamento não explicaria a aprendizagem, embora a motivação para aprender tenha sobre essa variável algum poder de predição. $\mathrm{O}$ estudo do autor evidencia a diferença entre os conceitos de expectativa pré-treinamento e de motivação para aprender e analisa que ainda há poucos estudos sobre expectativas pré-treinamento.

A variável crenças sobre o sistema de T\&D também se aproxima da variável expectativa pré-treinamento. Embora a primeira seja mais genérica para as ações de capacitação como um todo, a segunda está mais focada no que o participante espera daquele curso específico. Vale destacar dois estudos que abordam a predição das crenças sobre os resultados de treinamento. O de Freitas e Borges-Andrade (2004), que encontraram associação positiva entre as crenças e o impacto do treinamento no trabalho, e o de Marins (2008), que encontrou relacionamento positivo entre as crenças relativas ao treinamento e os resultados de aprendizagem.

No que diz respeito à reação ao treinamento, os resultados apresentam valores elevados, o que se deve, possivelmente, a várias razões. A primeira seria o alcance dos objetivos mais imediatos do treinamento - atendimento das expectativas dos treinandos —, que apresentou índices elevados. Os cursos são projetados para atender demandas organizacionais e necessidades individuais dos funcionários e o fato de os respondentes terem gostado do curso sugere que as técnicas e estratégias adotadas no treinamento deram os resultados esperados na percepção dos participantes. A segunda razão que pode ter contribuído para uma reação muito favorável ao curso é o vínculo que normalmente ocorre entre instrutores e treinandos. Esse vínculo ao final 
do curso, quando são coletadas as avaliações de reação, ainda é muito forte e as pessoas estão envolvidas com o poder sedutor que normalmente o saber promove. Abbad e colaboradores (2000) também encontraram resultados elevados para os itens que avaliam o entrosamento com instrutor e, para esses autores, esse resultado é questionável pelo fato de serem altamente influenciados por características pessoais. Outra possibilidade é que o fenômeno da desejabilidade social influencie a avaliação de reação, uma vez que ela ainda é preenchida no ambiente de sala de aula.

Pode-se, ainda, atribuir os índices elevados na avaliação de reação ao fato de os treinamentos oferecerem aos funcionários momentos de prazer no trabalho, uma vez que os profissionais que participam dos cursos o fazem voluntariamente e a área de T\&D busca despertar neles maior interesse em relação às atividades de capacitação.

Ainda em relação à reação ao treinamento, os resultados do presente estudo indicam que reação tem poder explicativo sobre a variável impacto do treinamento no trabalho. Assim, atesta que indivíduos satisfeitos com os cursos tendem a perceber melhor aplicação nas tarefas do trabalho dos conteúdos aprendidos. A satisfação provoca nas pessoas, de modo geral, atitudes mais positivas. No caso de treinandos, essas atitudes positivas contribuem para a execução das tarefas do trabalho. Embora Alliger e Janak (1989) e Abbad e colaboradores (2000) questionem a relação entre os níveis de resultados de treinamento proposta por Kirkpatrick (1976), estudos como o de Abbad e colaboradores (2000) e o de Lacerda (2002) demonstram que, pelo menos entre esses dois níveis - reação e impacto - , as relações tendem a realmente ser positivas.

A hipótese que previa que os cursos de conteúdo cognitivo apresentariam melhor percepção de impacto foi confirmada. Esse resultado pode ser atribuído ao fato de que a aplicação de conhecimentos de conteúdo cognitivo é mais fácil de mensurar pelo próprio participante do que a aplicação de conteúdos comportamentais. A percepção de impacto no treinamento de conteúdos comportamentais ficaria mais evidente em médio e longo prazo.

Outra explicação a se considerar seria a possível dificuldade das pessoas que foram treinadas em admitir que, após o curso, apresentaram mudanças em seu comportamento. Ou seja, pode existir por parte das pessoas maior resistência ou dificuldade para aplicar seus conteúdos ou mesmo para admitir os possíveis benefícios gerados. A aplicação desses conteúdos refletiria mudanças de comportamentos muitas vezes cristalizados. Ao passo que a aplicação no trabalho de conteúdos cognitivos depende de ferramentas mais fáceis, ou seja, aplicação correta de legislação, utilização de softwares etc. Outros pontos favoráveis à aplicação mais imediata dos cursos chamados cognitivos são 
a necessidade urgente de respostas às demandas de trabalho e a possibilidade de a aplicação depender exclusivamente do treinando. Os cursos comportamentais, muitas vezes para produzirem um resultado melhor, devem atender à equipe de trabalho como um todo. Não adiantaria melhorar, por exemplo, o nível do atendimento a clientes de apenas uma pessoa do setor. Também não traria resultados satisfatórios o treinamento de apenas um funcionário numa equipe com problemas de relacionamento.

Assim, numa análise global, conclui-se que a hipótese 2 foi confirmada, como pode ser visto na figura.

Figura

Modelo empírico para impacto do treinamento no trabalho

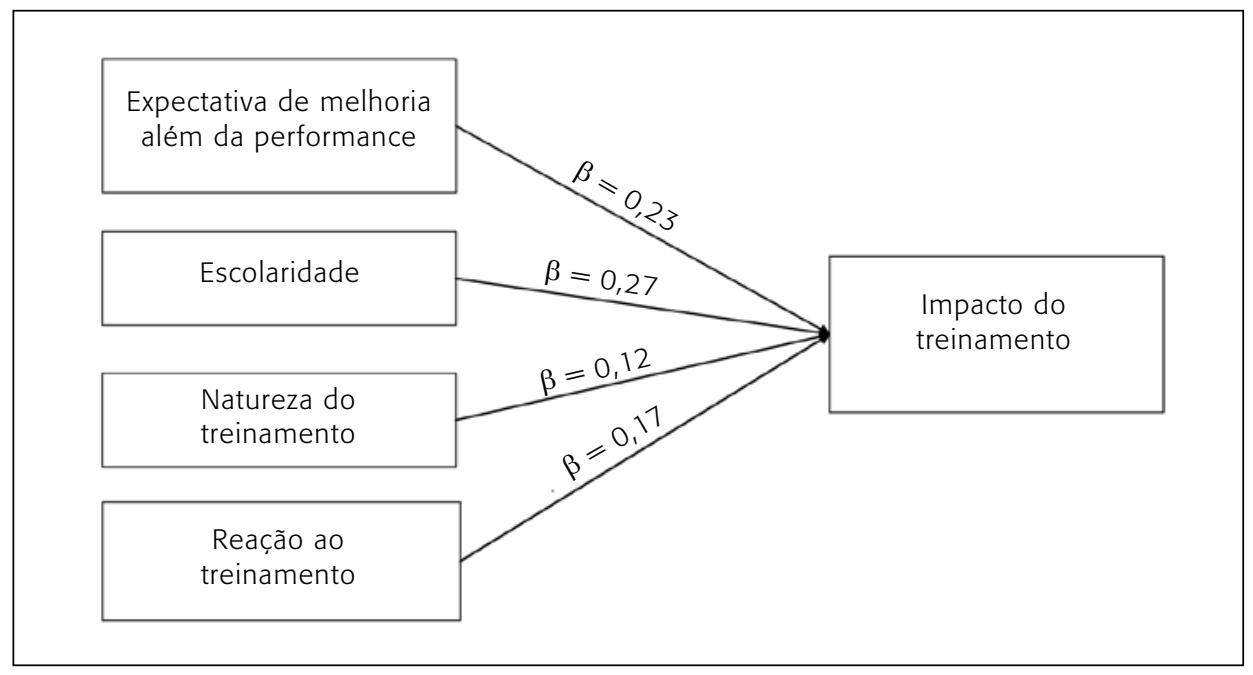

\section{Conclusão}

O treinamento conseguiu alcançar seu espaço, provando sua importância na estratégia organizacional. A avaliação precisa ser vista como parte integrante do programa de treinamento para reforçar sua importância. Cabe à avaliação oferecer às organizações resultados que auxiliem no processo decisório para contemplar melhorias na gestão de pessoas. A necessidade de alto grau de competência dos empregados é essencial para conduzir a organização a patamares de excelência. Portanto, as avaliações da eficácia e da efetividade do treinamento aceleram as possibilidades de feedback que as organizações tanto precisam. 
O presente estudo cumpriu o objetivo de realizar uma avaliação de treinamento, contemplando os níveis de reação e de impacto. Além disso, foi incluída no modelo de pesquisa a variável expectativa para treinamento, que vem atender lacuna existente na avaliação de características que podem contribuir na efetividade do treinamento. As expectativas dos treinandos e outras características de conteúdo motivacional permitem aos profissionais de T\&D investirem em ações que potencializem os resultados esperados nos treinamentos.

Os resultados dessa pesquisa apontam que expectativas otimistas relativas a melhorias que vão além da aplicação imediata nas tarefas cotidianas de trabalho contribuem para resultados favoráveis no impacto do treinamento no cargo. Assim, as organizações devem investir na divulgação e disseminação dos seus cursos como forma de despertar expectativas e, consequentemente, ampliar o impacto dos cursos realizados. Para Ribeiro (2000), as atitudes e as motivações são mais fáceis de alterar que as capacidades, o que reflete a importância de se identificar as expectativas como preditoras do impacto do treinamento no trabalho.

A pesquisa trouxe novos resultados acerca de variáveis que predizem os resultados de treinamento, confirmando achados de estudos anteriores, bem como refutando outros. O fato de alguns resultados apresentados confirmarem estudos anteriores e outros contestarem demonstra a necessidade de continuar investigando variáveis que interferem positivamente ou inibem os resultados esperados nas ações de capacitação.

Os bons resultados obtidos para a variável impacto do treinamento no trabalho traduzem um cenário otimista, sinalizando que os programas de capacitação da instituição pesquisada foram bem elaborados, contemplando as reais necessidades dos funcionários e as demandas da organização. A mensuração da transferência da aprendizagem ocorrida durante os treinamentos para as situações de trabalho oferece resultado em médio ou longo prazo e caracteriza o impacto que o treinamento produziu.

Como contribuições mais relevantes deste estudo, pode-se destacar a inclusão da variável expectativa de treinamento no rol de possíveis preditores dos resultados de ações de capacitação, além da ampliação das variáveis que podem explicar o impacto do treinamento no trabalho. Vale destacar, também, que as implicações teóricas e práticas decorrentes dos resultados alcançados na presente pesquisa podem contribuir para o planejamento de instituições que investem em T\&D.

Finalmente, cabe analisar que o presente projeto vem atender a uma parte da agenda proposta por Borges-Andrade e Oliveira-Castro (1996) no 
que diz respeito, principalmente, à criação de modelos preditivos, integrando os diversos níveis de avaliação.

As limitações do estudo ficaram principalmente por conta da restrição da amostra, ou seja, funcionários públicos de apenas uma organização, o que reduz a possibilidade de generalizar os resultados. Ainda pode-se destacar como limitação do estudo a pouca variabilidade da carga horária dos cursos, o que impediu que fossem realizadas análises mais detalhadas a respeito das características dos treinamentos.

Como agenda de pesquisa para a área, propõe-se que sejam estudados resultados que envolvam outras características individuais como: a quantidade de anos de escolaridade, o tempo decorrido desde o último treinamento, o número de treinamentos de que já participou, variáveis motivacionais. Propõe-se também que sejam estudadas as mesmas variáveis em instituições privadas, bem como a distinção entre a avaliação de cursos ministrados internamente e de cursos externos para os quais os funcionários são encaminhados.

Considerando também os diversos resultados da literatura da área sobre a importância das variáveis situacionais como preditoras do impacto do treinamento no trabalho, seria interessante desenvolver novas pesquisas que avaliassem se nos diferentes ambientes dentro de uma mesma organização há maior ou menor transferência de treinamento para o trabalho.

\section{Referências}

ABBAD, G.S. Um modelo integrado de avaliação do impacto do treinamento no trabalho - Impact. Tese (doutorado) — Instituto de Psicologia, Universidade de Brasília, Brasília, 1999.

; BORGES-ANDRADE, J.E. Aprendizagem humana em organizações de trabalho. In: ZANELLI, J.C.; BORGES-ANDRADE, J.E.; BASTOS, A.V.B. (Org.). Psicologia, organizações e trabalho no Brasil. Porto Alegre: Artmed, 2004.

et al. Treinamento: análise do relacionamento da avaliação nos níveis de reação, aprendizagem e impacto no trabalho. Revista de Administração Contemporânea, v. 14, n. 3, p. 25-45, 2000.

ALLIGER, G.M.; JANAK, E.A. Kirkpatrick's levels of training criteria: thirty years later. Personnel Psychology, v. 42, n. 2, p. 331-342, 1989.

ALVAREZ, K.; SALAS, E.; GAROFANO, C.M. An integrated model of training evaluation and effectiveness. Human Resource Development Review, v. 3, n. 4, p. 385-416, 2004. 
BASTOS, A.V.B. O suporte oferecido pela pesquisa na área de treinamento. Revista de Administração da USP, v. 26, n. 4, p. 87-102, 1991.

BORGES-ANDRADE, J.E. Avaliação somativa de sistemas instrucionais: integração de três propostas. Tecnologia Educacional, v. 11, n. 46, p. 29-39, 1982.

. Desenvolvimento de medidas em avaliação de treinamento. Estudos de Psicologia, 7 (Número Especial), p. 31-43, 2002.

; OLIVEIRA-CASTRO, G. Treinamento no Brasil: reflexões sobre suas pesquisas. Revista de Administração, v. 31, n. 2, p. 112-125, 1996.

CAMPOS, K.C.L. et al. Avaliação do sistema de treinamento e desenvolvimento em empresas paulistas de médio e grande porte. Psicologia Reflexão e Crítica, v. 17, n. 3, p. 435-446, 2004

DORON, R.; PAROT, F. Dicionário de psicologia. 3. ed. São Paulo: Ática, 2001.

ENZMANN, D. RanEigen: a program to determine the parallel analysis criterion for the number of principal components. Applied Psychological Measurement, v. 21, n. 232, 1997.

FORD, J.K. et al. Factors affecting the opportunity to perform trained tasks on the job. Personnel Psychology, v. 45, n. 3, p. 511-527, 1992.

FREITAS, I.A.; BORGES-ANDRADE, J.E. Efeitos de treinamento nos desempenhos individual e organizacional. Revista de Administração de Empresas, v. 44, n. 3, p. 44-56. 2004.

GOLDSTEIN, I.L. Training in work organizations. In: DUNNETE, M.D.; HOUGH L. (Org.). Handbook of industrial and arganizational psychology. Palo Alto: Consulting Psychology Press, 1991. p. 507-619.

KIRKPATRICK, D.L. Evaluation of training. In: CRAIG R.L. (Org.). Training and development handbook. New York: Mc Graw-Hill, 1976. p. 18.1-18.27.

LACERDA, E.M. Avaliação do impacto do treinamento no trabalho: investigando variáveis motivacionais e organizacionais como suas preditoras. Dissertação (mestrado) — Instituto de Psicologia, Universidade de Brasília, Brasília, 2002.

LATHAM, G.P. Human resource training and development. Annual Review of Psychology, n. 39, p. 545-582, 1988.

MARINS J.L. Avaliação de treinamento nos níveis de aprendizagem e reação: a influência da motivação para aprender e das crenças sobre o sistema de treinamento. Dissertação (mestrado) — Universidade Salgado de Oliveira, Niterói, 2008. 
MEIRA, M. Disseminação de informações sobre treinamento: construção e validação de um instrumento de medida. Dissertação (mestrado) — Universidade de Brasília, Brasília, 2004.

MENARD, Scott. Applied logistic regression analysis. Thousand Oaks: Sage, 1995. (Series: Quantitative Applications in the Social Sciences, v. 106)

MENESES, P.P.M. Autoeficácia, lócus de controle, suporte à transferência e impacto de treinamento no trabalho. Dissertação (mestrado) - Universidade de Brasília, Brasília, 2002.

MYERS, C. Core skills and transfer in the youth training schemes: a field study of trainee motor mechanics. Journal of Organization Behavior, n. 13, p. 625-632, 1992.

NADLER, L. The handbook of human resources development. New York: Wiley 1984.

PILATI, R.; ABBAD, G.D.S. Análise fatorial confirmatória da escala de impacto do treinamento no trabalho. Psicologia: teoria e pesquisa, v. 21, p. 43-51, 2005.

RIBEIRO, C. Em torno do conceito locus de controlo. Revista Máthesis, n. 9, p. 297314, 2000.

ROWOLD, J. Individual influences on knowledge acquisition in a call center training context in Germany. International Journal of Training and Development, Oxford, v. 11, n. 1, p. 21-34, 2007.

SALAS, E.; CANNON-BOWERS, J.A. The science of training: a decade of progress. Annual Review Psychology, n. 52, p. 471-499, 2001.

SALLORENZO, L. H. Avaliação do impacto de treinamento no trabalho: analisando e comparando modelos de predição. Dissertação (mestrado) - Universidade de Brasília, Brasília, 2000.

TABACHNICK, B.G.; FIDELL, L.S. Using multivariate statistics. New York: HarperCollins College Publishers, 1996.

TZINER, A. et al. Effects of trainee characteristics on training effectiveness. International Journal of Selection and Assessment, Oxford, v. 15, n. 2, p. 167-174, 2007.

VARGAS, M.R.; ABBAD, G. Bases conceituais em treinamento, desenvolvimento e educação - T\&D. In: BORGES-ANDRADE, J.E.; ABBAD, G.S.; MOURÃO, L. (Org.). Treinamento, desenvolvimento e educação em organizações e trabalho: fundamentos para gestão de pessoas. Porto Alegre: Artmed. 2006. p. 137-158. 
VELADA, R. et al. The effects of training design, individual characteristics and work environment on transfer of training. International Journal of Training and Development, Oxford, v. 11, n. 4, p. 282-294, 2007.

VROOM, V.H. Work and motivation. New York: Wiley, 1964.

WARR, P.; BUNCE, D. Trainee characteristics and outcomes of open learning. Personnel Psychology, v. 48, n. 2, p. 347-375, 1995.

WEXLEY, K.N. Personnel training. Annual Review of Psychology, n. 35, p. 519-551, 1984. 